\title{
CORRECTION
}

\section{Correction: IQGAP1 promotes anoikis resistance and metastasis through Racl-dependent ROS accumulation and activation of Src/FAK signalling in hepatocellular carcinoma}

Chun-fen Mo, Jun Li, Shu-xia Yang, Hui-jie Guo, Yang Liu, Xing-yan Luo, Yan-tang Wang, Min-hui Li, Jing-yi Li and Qiang Zou British Journal of Cancer (2021) 124:1895; https://doi.org/10.1038/s41416-021-01355-4

Correction to: British Journal of Cancer (2020) 123, 1154-1163; https://doi.org/10.1038/s41416-020-0970-z, published online 7 July 2020

Since the publication of this paper, the authors have noticed that the Funding information is not complete and that the National Natural Science Foundation of China No. 81702446 project was not included. The complete Funding information is stated below, and the original article has been corrected. The authors apologise for this oversight.

\section{ADDITIONAL INFORMATION}

Funding information This study was supported by the National Natural Science Foundation of China (81402944, 81702446, 81871300), Application and Basic Project of Science and Technology Department of Sichuan Province (2018JY0440, 2017JY0174) and Research Fund of Chengdu Medical College (CYZ16-01). 